\title{
3 ANALYZING THE SAFETY OF WORKFLOW AUTHORIZATION MODELS
}

\author{
Wei-Kuang Huang and Vijayalakshmi Atluri
}

\begin{abstract}
Workflow Management Systems (WFMS) are being widely used today by organizations to coordinate the execution of various applications representing their day-to-day tasks. To ensure that these tasks are executed by authorized users or processes (subjects), and to make sure that authorized subjects gain access on the required objects only during the execution of the specific task, granting and revoking of privileges need to be synchronized with the progression of the workflow through proper authorization mechanisms. Recently, Atluri and Huang have proposed a workflow authorization model (WAM) that provides such synchronization. This paper, first extends WAM to support roles and authorization constraints such as separation of duties. Second, it develops methodologies to analyze the safety of workflow authorization model when authorization constraints are imposed. The analysis is carried out by modeling WAM as a suitable Petri net (PN) and by utilizing the well-established analysis techniques of PNs.
\end{abstract}

\subsection{INTRODUCTION}

Workflow Management has emerged as the technology to automate the coordination of day-to-day activities (called tasks) of business processes. Today wide use of Workflow Management Systems (WFMS) can be found in a number of domains including office automation, finance and banking, healthcare, telecommunications, manufacturing and production. The various tasks in a workflow are executed by several users or programs according to the organizational rules relevant to the processes represented by the workflow. To ensure that these tasks are executed by authorized users or processes (subjects), proper authorization mechanisms must be in place. Moreover, to make sure that authorized 
subjects gain access to the required objects only during the execution of the specific task, granting and revoking of privileges need to be synchronized with the progression of the workflow. Recently, Atluri and Huang have proposed a workflow authorization model (WAM) [2] that provides such synchronization.

It is common to many organizations to express security policies in terms of roles rather than users. For example, a nurse is authorized to administer a medication to a patient. Roles represent organizational agents intended to perform certain job functions within the organization. Users in turn are assigned appropriate roles based on their qualifications. Such role based authorization simplifies security administration. In addition, rules specifying separation of duties are imposed to reduce the risk of frauds by not allowing any individual to have sufficient authority within the system to perpetrate a fraud on his own. Such authorization constraints can be found in many application domains. For example, in a paper reviewing process, a person is never allowed to review his/her own paper, and a paper must be reviewed by at least three different individuals.

Although WAM is capable of providing the synchronization of authorization flow with the workflow and supports role-based authorization, it is not capable of supporting other essential requirements such as separation of duties. Although many research prototypes and commercial WFMS products are available today (e.g., Lotus Notes) that provide support for role-based authorization, they are not capable of modeling separation of duties. Since no proper support is provided, currently these constraints have to be implemented as ad hoc application code.

The significant work in this direction is due to Sandhu [10]. However, this research is not adequate to specify separation of duties in WFMS environment since it does not specify access control in terms of tasks. Recently, Bertino et al. [3], have identified several types of authorization constraints, including separation of duties. They have categorized constraints imposed on role and user assignments to tasks into three types: static, dynamic and hybrid constraints and have developed an approach to determine their consistency by first expressing the authorization constraints as clauses in a logic program. They have also proposed algorithms to check for the consistency of the constraints and to assign users and roles to tasks that constitute the workflow in a such a way that no constraints are violated. The primary emphasis of [3] is verification of the consistency of authorization constraints. However, since the predicates representing the constraints do not include the objects, it is not capable of distinguishing one workflow instance from the other. Thus, it is not capable of modeling inter-instance authorization constraints.

In this paper, we show how authorizations can be derived when constraints expressing separation of duties are enforced. This requires examining the current state of the set of authorizations as well as the state of the workflow. Our approach conducts a run-time evaluation of the state to authorize users to execute a task. The major distinction between our work and that proposed in [3] is that our WAM is capable of modeling separation of duties constraints on 
multiple workflow instances thus is able to capture inter-instance constraints. This is because, authorization constraints are specified consisting of authorizations themselves as predicates. This will also allow enforcement of constraints based on authorizations derived by other means, but not necessarily through the workflow executions.

In this paper, we also provide a formal model based on Petri nets by enhancing the Color-Timed Petri Net proposed in [2] to model role-based access control with separation of duties. Representing WAM as a Petri net allows one to visually depict the workflow behavior through its graphical representation and to analyze its behavior through its rich set of analysis techniques. Analysis helps one to understand the implications of the authorization policies. Although each policy may appear innocent in isolation, their cumulative effect may lead to an undesirable authorization state [11]. (See section 3.3 for a definition of authorization state.) So for a given initial authorization state and a set of security policies specified by authorization rules, analysis requires determining all the reachable authorization states. This, known as the safety problem, first identified by Harrison, Ruzzo and Ullman [6], specifically can be stated as the following question: "Is there a reachable state in which a particular subject possesses a particular privilege for a specific object?" We develop methodologies to analyze the safety of workflow authorization model when authorization constraints such as separation of duties are imposed.

\subsection{WORKFLOW AUTHORIZATION MODEL}

To ensure that authorized subjects gain access on the required objects only during the execution of the specific task, WAM synchronizes authorization flow with the workflow by synchronizing granting and revoking of privileges with the initiation and completion of the tasks. To achieve this synchronization, WAM associates an Authorization Template (AT) with each task, which specifies the static parameters of the authorization that can be defined during the design of the workflow. When a task starts its execution, this AT is used to derive the actual authorization. In this section, we review WAM with its extension to incorporate role based authorizations.

A workflow $W$ can be represented as a partially ordered set of tasks $\left\{t w_{1}, t w_{2}\right.$ $\left.\ldots t w_{n}\right\}$, where each task $t w_{i}$ in turn can be defined as a set $O P_{i}$ of a partial or total order of operations $\left\{o p_{1}, o p_{2} \ldots o p_{n}\right\}$ that involve manipulation of objects [5]. Processing of a task involves accessing certain objects by certain subjects with certain privileges. To execute a task $t w_{i}$, relevant privileges on required objects have to be granted to appropriate subjects.

Let $S=\left\{s_{1}, s_{2} \ldots\right\}$ denote the set of subjects, $O=\left\{o_{1}, o_{2} \ldots\right\}$ the set of objects $\Gamma=\left\{\gamma_{1}, \gamma_{2} \ldots\right\}$ the set of objects types and $R=\left\{r_{1}, r_{2} \ldots\right\}$ the set of roles. The function $F: O \rightarrow \Gamma$. That is, if $F\left(o_{i}\right)=\gamma_{j}$, then $o_{i}$ is of type $\gamma_{j}$. $G: S \rightarrow R$. I.e., if $G\left(s_{i}\right)=r_{j}$, then $s_{i}$ is of role $r_{j}$. Let $P R$ denote a finite set of privileges. We use $S_{r_{i}}$ to denote the set of subjects that belong to role $r_{i}$ and $O_{\gamma_{i}}$ to denote the set of objects of type $\gamma_{i}$. 
Definition 1 1. Time set $\mathcal{T}=\{\tau \in \mathcal{R} . \mid \tau \geq 0\}$

2. a time interval $\left\{\left[\tau_{l}, \tau_{u}\right] \in \mathcal{T} \times \mathcal{T} \mid \tau_{l} \leq \tau_{u}\right\}$ represents the set of all closed intervals.

According to definition 1 an interval is defined by its lower and upper bounds, $\tau_{l}$ and $\tau_{u}$, respectively, where each of $\tau_{l}$ and $\tau_{u}$ can either be a constant or an expression.

Definition 2 A task $t w_{i}$ is defined as $\left(O P_{i}, \Gamma_{I N_{i}}, \Gamma_{O U T_{i}},\left[\tau_{l_{i}}, \tau_{u_{i}}\right]\right)$, where $O P_{i}$ is the set of operations to be performed in $t w_{i}, \Gamma_{I N_{i}} \subseteq \Gamma$ is the set of object types allowed as inputs, $\Gamma_{O U T_{i}} \subseteq \Gamma$ is the set of object types expected as outputs, and $\left[\tau_{l_{i}}, \tau_{u_{i}}\right]$ is the time interval during which $t w_{i}$ must be executed.

Here $\left[\tau_{l_{i}}, \tau_{u_{i}}\right]$ specifies the temporal constraint stating the lower and upper bounds of the time interval during which a task is allowed to be executed.

Definition 3 A task-instance tw-inst ${ }_{i}$ is defined as: $\left(O P E R_{i}, I N_{i}, O U T_{i}\right.$, $\left.\left[\tau_{s_{i}}, \tau_{f_{i}}\right]\right)$ where $O P E R_{i}$ is the set of operations performed during the execution of $t w_{i}, I N_{i}$ is the set of input objects to $t w_{i}$ such that $I N_{i}=\{x \in O \mid F(x) \in$ $\left.\Gamma_{I N_{i}}\right\}, O U T_{i}$ is the set of output objects from $t w_{i}$ such that $O U T_{i}=\{x \in$ $\left.O \mid F(x) \in \Gamma_{O U T_{i}}\right\}$, and $\left[\tau_{s_{i}}, \tau_{f_{i}}\right]$ is the time interval during which $t w_{i}$ has been executed.

Whenever a task is executed, a task-instance will be generated. Thus, a task $t w_{i}$ may generate several tw-inst ${ }_{i}$ 's. $\tau_{s_{i}}$ and $\tau_{f_{i}}$ in the above definition indicate the time at which that particular task-instance has started and finished execution, respectively, whereas $\left[\tau_{l_{i}}, \tau_{u_{i}}\right]$ represent the time during which the task is allowed to be executed. Note that $\left[\tau_{l_{i}}, \tau_{u_{i}}\right]$ may differ from $\left[\tau_{s_{i}}, \tau_{f_{i}}\right]$. However, to ensure the temporal constraints, $\left[\tau_{s_{i}}, \tau_{f_{i}}\right]$ must be within $\left[\tau_{l_{i}}, \tau_{u_{i}}\right]$.

Definition 4 An authorization is a 4-tuple $A=\left(s, o, p r,\left[\tau_{b}, \tau_{e}\right]\right)$, where subject $s$ is granted access on object $o$ with privilege $p r$ at time $\tau_{b}$ and is revoked at time $\tau_{e}$.

An authorization base $A B=\left\{A_{1}, A_{2} \ldots\right\}$ is a finite set of authorizations. As workflow execution progresses, all authorizations that have been generated are added to the set $A B$.

Definition 5 Given a task $t w_{i}$, an authorization template $A T\left(t w_{i}\right)$ is defined as a 4-tuple $A T\left(t w_{i}\right)=\left(\left(r_{i},-\right),\left(\gamma_{i},-\right), p r_{i},\left[\tau_{l_{i}}, \tau_{u_{i}}\right]\right)$ where

(i) $\left(r_{i},-\right)$ is a subject hole which can be filled by a subject $s_{i}$ where $G\left(s_{i}\right)=r_{i}$,

(ii) $\left(\gamma_{i},-\right)$ is an object hole which can be filled by an object $o_{i}$ where $F\left(o_{i}\right)=\gamma_{i}$,

(iii) $p r_{i}$ is the privilege to be granted to $s_{i}$ on object $o_{i}$.

(iv) $\left[\tau_{l i}, \tau_{u i}\right]$ is the time interval during which the task must be executed.

In the definition for $A T\left(t w_{i}\right)$ (i) says that only subjects belonging to role $r_{i}$ is allowed to execute $t w_{i}$ thus the subject hole $\left(r_{i},-\right)$ allows only subjects that belong to role $r_{i}$, (ii) dictates that only objects of type $\gamma_{i}$ can be processed by 
$t w_{i}$, thus the object hole $\left(\gamma_{i},-\right)$ allows objects of only type $\gamma_{i}$, (iii) says that a subject requires a privilege $p r_{i}$ on the objects that arrive at $t w_{i}$ for processing, and (iv) says the default interval for the authorization template will be the valid time interval for the task.

Authorization templates are attached to the tasks in a workflow. A task $t w_{i}$ may have more than one authorization template associated with it. More $A T$ s are required in cases where there are more than one type of object to be processed, or more than one subject is required to perform the processing. To distinguish the privileges in $A T$ from those in $A$, we often use $\operatorname{pr}(A T)$ to denote the privilege component of an authorization template $A T$. An authorization template enables one to specify rules such as "Only a clerk is allowed to perform check preparation during time 10 and 50." These can actually be stated during the design process by the workflow designer.

\section{Definition 6 [Authorization Derivation Rule]}

Given an authorization template $A T\left(t w_{i}\right)=\left(\left(r_{i},-\right),\left(\gamma_{i},-\right), p r_{i},\left[\tau_{l i}, \tau_{u i}\right]\right)$ of task $t w_{i}$, an authorization $A_{i}=\left(s_{i}, o_{i}, p r_{i},\left[\tau_{b_{i}}, \tau_{e_{i}}\right]\right)$ is derived as follows:

Grant Rule: Suppose object $x$ is sent to subject $y$ at $\tau_{a_{i}}$ to start $t w_{i}$. If $x \in O_{\gamma i}$ and $y \in S_{r i}$ and $\tau_{a_{i}} \leq \tau_{u_{i}}$,

$s_{i} \leftarrow y, o_{i} \leftarrow x, p r_{i} \leftarrow p r(A T)$;

$\tau_{e_{i}} \leftarrow \tau_{u_{i}}$

if $\tau_{a_{i}} \leq \tau_{l_{i}}, \tau_{b_{i}} \leftarrow \tau_{l_{i}}$; otherwise $\tau_{b_{i}} \leftarrow \tau_{a_{i}}$.

Revoke Rule: Suppose $t w_{i}$ ends at $\tau_{f_{i}}$ at which point $x$ leaves $t w_{i}$.

If $\tau_{f_{i}} \leq \tau_{u_{i}}, \tau_{e_{i}} \leftarrow \tau_{f_{i}}$.

Example 1 We explain now how authorizations are derived from the authorization templates with an example. Considering a check processing example [2] consisting of three tasks $t w_{1}, t w_{2}$ and $t w_{3}$ denoting prepare check, approve check and issue check, respectively. They can be expressed as follows:

$t w_{1}=(\{$ prepare check $\},\{$ check $\},[10,50])$

$t w_{2}=(\{$ approve check $\},\{$ check $\},\{$ check $\},[20,60])$

$t w_{3}=(\{$ issue check $\},\{$ check $\},\{$ check $\},[40,80])$

Suppose the associated roles for performing these processes are clerk, manager and clerk, respectively. Assume Mary and John are clerks and Peter is the only manager. We define the following authorization templates.

$A T\left(t w_{1}\right)=(($ clerk, -$),($ check,-), prepare, $[10,50])$

$A T\left(t w_{2}\right)=(($ manager,-),$($ check,-), approve, $[20,60])$

$A T\left(t w_{3}\right)=(($ clerk,,$),($ check, -$)$, issue, $[40,80])$

Now suppose the check $c k 1$ for payment arrives at time 40 and John starts $t w_{1}$. Both subject and object are filled into the authorization template $A T\left(t w_{1}\right)$, generating an authorization (John, ck1, prepare, [40,50]). Suppose John finishes $t w_{1}$ at 47 , then the authorizations on $c k 1$ are revoked for John by replacing the upper bound with 47, thus forming the authorization (John, ck1, prepare, $[40,47])$. Similarly, other authorizations will be derived as approve and issue tasks are executed. 


\subsection{WAM WITH SEPARATION OF DUTIES}

Separation of duties can be expressed as constraints. In [3], Bertino et al. have identified several types of authorization constraints, including separation of duties. Similarly, we also express separation of duties as rules.

Definition 7 Given an authorization template $A T\left(t w_{i}\right)=\left(\left(r_{i},-\right),\left(\gamma_{i},-\right), p r_{i}\right.$, $\left.\left[\tau_{l_{i}}, \tau_{u_{i}}\right]\right)$, we define a set of potential authorizations, $P A_{i}$, representing all possible authorizations that can be potentially derived from $A T\left(t w_{i}\right)$. Each potential authorization $p a$ in $P A_{i}$ is a triple $\left(s_{i}, o_{i}, p r_{i}\right)$ such that $s_{i} \in S_{r_{i}}, o_{i} \in O_{\gamma_{i}}$.

Definition 8 Given an authorization $A=\left(s, o, p r,\left[\tau_{b}, \tau_{e}\right]\right)$ in $A B$, we define a non-temporal projection $A_{N T}$ of $A$ as $A_{N T}=(s, o, p r)$. The non-temporal projection of $A B, A B_{N T}=\left\{A_{N T_{1}}, A_{N T_{2}} \ldots\right\}$.

In our formalism, we assume each constraint $c_{i}$ is a logical expression of the form: $q \leftarrow p$ where $p$ is any logical expression consisting of $A_{N T}$ as literals and $q$ is a single literal which is always either $p a$ or $\sim p a$ such that $p a \in P A_{i}$ of some $t w_{i}$. We also denote $s(p)$ (or $s(q)$ ) as the set of subjects that are specified in $p a \in P A_{j}$ (or $p a \in P A_{i}$ ). Enforcement of a constraint would either force or prohibit assignment of a specific user to a task. Therefore, separation of duties constraints fall into two categories. Note that this categorization is different from that of [3].

- Exclusive type: In this type, $q$ is always of the form $\sim p a$ where $p a \in P A_{j}$ for some $t w_{j}$.

- Assertive type: In this type, $q$ is always of the form $p a$ where $p a \in P A_{j}$ for some $t w_{j}$.

As an example of an exclusive constraint, consider once again the check processing example introduced in section 3.2. Suppose the business policy of the bank is such that it does not allow any single individual to both prepare and issue a check. This policy can be expressed as constraint $c_{1}$ : A clerk who prepares a check cannot issue the same check. Formally, $c_{1}$ can be expressed as follows.

$c_{1}:\left(\forall x \in S_{\text {clerk }}, y \in O_{\text {check }}\right) \cdot(\sim(x, y$, issue $) \leftarrow(x, y$, prepare $))$

On the other hand, if the business policy states that a check can be issued only by an individual who prepares it. This can be formally stated as:

$c_{2}:\left(\forall x \in S_{\text {clerk }}, y \in O_{\text {check }}\right) \cdot((x, y$, issue $) \leftarrow(x, y$, prepare $)$

Thus $c_{2}$ is an assertive type constraint. Based on whether a constraint is either assertive or exclusive type, certain users in the role of clerks are not eligible to execute the task of issuing a check. Thus, the set of eligible users for each task changes dynamically based on the current state of the authorization base. Moreover, the eligible users vary from one task-instance to another. Furthermore, only certain constraints play a role in deciding the eligibility of subject to execute a task. For example, $c_{1}$ affects the eligible set of subjects of $t w_{3}$ but not of $t w_{1}$ or $t w_{2}$ in our check processing example. Therefore we determine first the set of relevant constraints for each task $t w_{i}$, denoted as $C_{t w_{i}}$. 
Definition 9 We define $C_{t w_{i}}$ as follows: $C_{t w_{i}}=\left\{c_{j} \mid c_{j} \in C\right.$ which is of the form $q_{i} \leftarrow p_{j}$ and $\left.q_{i} \in P A_{i}\right\}$.

Then, for each task, we define a set of eligible subjects, denoted as $S_{i}^{e}(o)$ with respect to object $o$.

Definition 10 Given an authorization template $A T\left(t w_{i}\right),\left(\left(r_{i},-\right),\left(\gamma_{i},-\right), p r_{i}\right.$, $\left.\left[\tau_{l_{i}}, \tau_{u_{i}}\right]\right)$, we define the set of eligible subjects $S_{i}^{e}(o)$ as follows:

1. $S_{i}^{e}(o)=S_{r_{i}}$ if $C_{t w_{i}}=\emptyset$

2. $S_{i}^{e}(o)=S_{r_{i}}-s\left(q_{i}\right)$ if $c_{i}: q_{i} \leftarrow p_{j} \in C_{t w_{i}}$ is an exclusive constraint and $p_{j}$ is true with respect to $A B_{N T}$

3. $S_{i}^{e}(o)=s\left(q_{i}\right)$ if $c_{i}: q_{i} \leftarrow p_{j} \in C_{t w_{i}}$ is an assertive constraint and $p_{j}$ is true with respect to $A B_{N T}$

The above definition says that if the constraint specifying the separation of duties is of exclusive type, the set of eligible subjects is obtained by subtracting the disallowed subjects from the set of subjects playing the role assigned to execute the task. On the other hand, if the constraint is of assertive type, the set of eligible subjects is simply the set of subjects specified in $p a \in q$. If no constraints affect the task, then the set of eligible subjects is same as the set of subjects playing the role. An appropriate authorization must be generated from the authorization template at run time in such a way that the subject to execute the task must be chosen from the set of eligible subjects and only when the task receives an object with type specified in the authorization template. The following authorization derivation rule ensures this requirement.

Definition 11 [Authorization Derivation Rule for extended WAM] Given an authorization template $A T\left(t w_{i}\right)=\left(\left(r_{i},-\right)\left(\gamma_{i},-\right), p r_{i},\left[\tau_{l i}, \tau_{u i}\right]\right)$ of task $t w_{i}$, an authorization $A_{i}=\left(s_{i}, o_{i}, p r_{i},\left[\tau_{b_{i}}, \tau_{e_{i}}\right]\right)$ is derived as follows:

Grant Rule: Suppose object $x$ is sent to subject $y$ at $\tau_{a_{i}}$ to start $t w_{i}$. If $x \in O_{\gamma i}$ and $y \in S_{i}^{e}(x)$ and $\tau_{a_{i}} \leq \tau_{u_{i}}$,

$s_{i} \leftarrow y, o_{i} \leftarrow x, p r_{i} \leftarrow p r(A T)$;

$\tau_{e_{i}} \leftarrow \tau_{u_{i}}$

if $\tau_{a_{i}} \leq \tau_{l_{i}}, \tau_{b_{i}} \leftarrow \tau_{l_{i}}$; otherwise $\tau_{b_{i}} \leftarrow \tau_{a_{i}}$.

Revoke Rule: Suppose $w_{i}$ ends at $\tau_{f_{i}}$ at which point $o_{i}$ leaves $t w_{i}$.

If $\tau_{f_{i}} \leq \tau_{u_{i}}, \tau_{e_{i}} \leftarrow \tau_{f_{i}}$.

Note that the only difference between the above definition and earlier derivation rule in definition 6 is the set from which a subject is chosen. Without the separation of duties constraints, a subject is chosen from the set of subjects playing the specified role, whereas with separation of duties constraints, a subject is chosen from the set of eligible subjects which may be a subset of the previous set.

Definition 12 Given a workflow $W$, we define a workflow authorization state $A^{s}(W)$ as the set of current non-temporal projection of authorizations derived during the execution of $W$. 
For instance, in example 1, a workflow authorization state $A^{s}(W)$ could be $\{($ John, ck2 , prepare $),($ Peter, ck1, approve $)\}$, meaning that an authorization is granted to John for preparing $c k 2$ and to Peter to approve ck1. In the following, we explain the process of deriving authorizations by taking an example.

Example 2 Consider once again example 1. Suppose a separation of duties constraint is specified as $\left(\forall x \in S_{\text {clerk }}, y \in O_{\text {check }}\right) \cdot(\sim(x, y, i s s u e) \leftarrow$ $(x, y$, prepare $))$. Since $S_{1}^{e}(c k 1)=\operatorname{subject}($ clerk $)=\{$ John, Mary $\}$, Mary $\epsilon$ $S_{1}^{e}(c k 1)$, and $c k 1 \in O_{\text {check }}$, after the execution of task $t w_{1}$, an authorization (John, ck1, prepare, [40,47]) is generated. Similarly, after the execution of task $t w_{2}$, an authorization (Peter, ck1, approve, [47,54]) is generated. As a result of the constraint, the eligible set of subjects authorized to execute $t w_{3}$ are evaluated as follows: $S_{3}^{e}(c k 1)=S_{\text {clerk }}-\{s($ John, ck1$, prepare $)\}=\{$ Mary,$J o h n\}-\{J o h n\}=\{$ Mary $\}$. In other words, only Mary is allowed to execute $t w_{3}$. Thus the separation of duties constraint is satisfied.

\subsection{SAFETY ANALYSIS OF WAM}

In this section, we first present the Petri net representation of WAM through which we perform the safety analysis. Then we present the algorithm to test for the safety of WAM. We also report the implementation status of this module.

\subsubsection{Color Timed Petri Net (CTPN) - A Model to Represent Workflow Authorization Model}

In this section, we present the Color Timed Petri Net (CTPN) Model [2] to represent the WAM proposed in the previous section. Our CTPN is an extension of colored and timed Petri nets [7], which in turn are extensions of an ordinary Petri net.

Definition 13 A Color Timed Petri Net (CTPN) is a tuple $C T P N=(P N, \Sigma$, $C R, E, I N, D, t s)$ where

1. $P N=(P, T, F, M)$ is an ordinary Petri net.

2. $\Sigma=\left\{\sigma_{1}, \sigma_{2}, \ldots\right\}$ is a finite set of colors (or types),

3. $C R$ is a color function such that $C R(p) \subseteq \Sigma$, and $C R(m(p)) \subseteq C R(p)$,

4. $E$, the arc function such that: $\forall f(p, t), f(t, p) \in F, E_{f} \subseteq C R(p)_{M S}$,

5. $I N$ is an interval function associated with a transition, i.e., $I N: T \rightarrow$ $\mathcal{T} \times \mathcal{T}$ such that $I N\left(t_{i}\right)=\left[\tau_{l i}, \tau_{u i}\right]$ where $\left[\tau_{l i}, \tau_{u i}\right] \in \mathcal{T} \times \mathcal{T}$ and $t_{i} \in T$,

6. $D$ is a delay function associated to a place. i.e., $D: P \rightarrow \mathcal{T} \times \mathcal{T}$ such that $D\left(p_{j}\right)=\left[\delta_{j}^{m}, \delta_{j}^{M}\right]$, where $\left[\delta_{j}^{m}, \delta_{j}^{M}\right] \in \mathcal{T} \times \mathcal{T}$ and $p_{j} \in P$, and

7. $t s$ is a timestamp function such that $t s\left(m\left(p_{k}\right)\right)=\alpha_{k} \in \mathcal{T}$, which denotes the arrival time of the token to $p_{k}$.

We represent a token as $(v, x)$ where $v$ is the color of the token and $x$ the timestamp. Whenever a token moves from one place to another through a fired transition, its timestamp is modified to the firing time of the transition. 
We use $m(p)$ to denote the marking of place $p . m(p)$ is expressed as a multisets of tokens with respect to distinct colors. For example, $m(p)=g+r$ represents place $p$ containing a token of color $g$ and a token of color $r$, i.e., $C R(m(p))=\{g, r\}$.

The above definition dictates that each token has a color which is defined in the color set $\Sigma$. Each place has a color set (i.e., denoted as $C R(p)$ ) attached to it which specifies the set of allowable colors of the tokens to enter the place. For a token to reside in a place, it must satisfy that the color of token is a member in the color set of the place. Each arc $f(p, t)$ or $f(t, p)$ is associated with a color set such that this set is contained in the multi-sets of $C R(p)$. A transformation of colors may occur during firing of a transition. The firing of a transition is determined by the firing rules and the transformation by the arc function $E$. The firing rules can be formally stated as follows:

Definition 14 Given a transition $t_{i}$ such that $I N\left(t_{i}\right)=\left[\tau_{l i}, \tau_{u i}\right], \forall p_{j} \in \bullet t_{i}$ and $\forall p_{k} \in t_{i} \bullet$, for any $p_{j}$ marked with tokens $\left(v_{j 1}, x_{j 1}\right),\left(v_{j 2}, x_{j 2}\right) \ldots\left(v_{j n}, x_{j n}\right)$,

1. $\left(v_{j l}, x_{j l}\right)$ is said to be available only during the interval $\left[\delta_{j}^{m}+x_{j l}, \delta_{j}^{M}+x_{j l}\right]$,

2. $t_{i}$ is said to be enabled at time $x$ if $E_{f\left(p_{j}, t_{i}\right)} \leq m\left(p_{j}\right)$ and all tokens in $p_{j}$ are available at time $x$.

3. an enabled $t_{i}$ is firable if $\left.\max \left\{\left(\delta_{j}^{m}+x\right) \mid p_{j} \in \bullet t_{i}\right\} \leq \tau_{u i}\right) \wedge \min \left\{\left(\delta_{j}^{M}+x \mid p_{j} \in \bullet t_{i}\right\} \geq \tau_{l i}\right)$ is true. A firable transition may fire any time during the firable interval, $\left[\max \left\{\tau_{l i}, \max \left\{\delta_{j}^{m}+x \mid p_{j} \in \bullet t_{i}\right\}\right\}, \min \left\{\tau_{u i}, \min \left\{\delta_{j}^{M}+x \mid p_{j} \in \bullet t_{i}\right\}\right\}\right]$.

4. Suppose $t_{i}$ fires at $\tau_{i}$. Firing of $t_{i}$ results in a new marking $M^{\prime}$ as follows: $m^{\prime}\left(p_{k}\right)=m\left(p_{k}\right)+E_{f\left(t_{i}, p_{k}\right)}$ and $m^{\prime}\left(p_{j}\right)=m\left(p_{j}\right)-E_{f\left(p_{j}, t_{k}\right)}$ and the timestamp of each element in $m^{\prime}\left(p_{k}\right)$ is $\tau_{i}$.

A transition $t_{i}$ is enabled only if all of its input places $p_{j}$ contain at least as many available tokens of the type as that specified in the arc function $E_{f\left(p_{j}, t_{i}\right)}$ of the corresponding $f\left(p_{j}, t_{i}\right)$.

The delay associated with a place represents minimum $\delta^{m}(p)$ and maximum $\delta^{M}(p)$ delay a token is required to remain in that place after its arrival. The delay can be a constant $d$ where $\delta^{m}(p)=\delta^{M}(p)$. A token is said to be available only after the delay $D\left(p_{j}\right)$ has elapsed. On the other hand, the time interval associated with a transition states that it can fire only during this interval, irrespective of the tokens' timestamps in its input places. A transition is said to be enabled only if each of its input places has an available token.

A transition $t_{i}$ fires only if its enabling time falls within the specified time interval $I N\left(t_{i}\right)$. When more than one input place exists, the transition fires after the maximum delay of all the input places has elapsed. Both the time interval and the delay can be specified as variables instead of fixed values. Upon firing, a transition $t_{i}$ consumes as many tokens of colors from each of its input places $p_{j}$ as those specified in the corresponding $E_{f\left(p_{j}, t_{i}\right)}$ and deposits as many tokens with specified colors into each output place $p_{k}$ as those specified in the corresponding $E_{f\left(t_{i}, p_{k}\right)}$. That is, the arc function of $f\left(p_{k}, t_{i}\right)$ specifies the number of tokens of specified colors to be removed from $p_{j}$ when $t_{i}$ fires, 
and the arc function $f\left(t_{i}, p_{k}\right)$ specifies the number of tokens of specified colors to be inserted into $p_{k}$ when $t_{i}$ fires.

\subsubsection{CTPN Representation of WAM}

In the following, we illustrate how each component of WAM is represented in the CTPN. Given a task $t w$ with $A T(t w)=\left((r,-),(\gamma,-), p r,\left[\tau_{l}, \tau_{u}\right]\right)$, execution of $t w$ generates an authorization $A=\left(s, o, p r,\left[\tau_{b}, \tau_{e}\right]\right)$.

(1) A role $r$ is represented as a place with an associated color set that contains all subjects. (2) A object type $\gamma$ is represented as a place with a color set that contains all objects. (3) A subject, an object and a (subject, object) pair are represented by a token with respective color. (4) A subject assigned to a role $r$ is represented as a token deposited in a place $r$. (5) An object of type $\gamma$ is represented as a token in place $\gamma$. (6) A privilege $p r$ to perform the task is represented by a place with a color set expecting tokens of (subject, object) type. An arc is connected from grant transition to the privilege place and another from the privilege place to the revoke transition with the arc function of expected (subject, object) pair. (7) The grant and revocation processes are represented as input (grant) and output (revoke) transitions of the place representing the privilege, respectively. (8) A subject hole $(r,-)$ is represented as an input arc from place $r$ to the grant transition with an arc function specifying the subjects of role $r$. (9) An object hole $(\gamma,-)$ is represented as an input arc from place $\gamma$ to the grant transition with an arc function specifying the objects of type $\gamma$. (10) The time interval $\left[\tau_{l}, \tau_{u}\right]$ associated with the grant or revoke transitions denotes the specified interval during which the authorization is valid. (11) An authorization corresponds to a filled privilege place. (12) The time interval $\left.\left[\tau_{b}, \tau_{e}\right]\right)$ in an authorization is the time interval during which a token resides in place $p r$ (the difference between $\tau_{b}$ and $\tau_{e}$ is the duration for executing tw, denoted as $D(p r))$. (13) A constraint $c: q_{i} \leftarrow p_{j} \in C_{t w_{i}}$ can be represented by a subnet as follows: For each $A_{j}$ in $p_{j}$ create a place $C$ with an input arc $\left(f_{1}\right)$ from the grant transition of $p r_{j}$ in $p_{j}$ and an output arc $\left(f_{2}\right)$ to the grant transition of $p r_{i}$ in $q_{i}$, both with arc function $(x, o)$ where $x$ and $o$ are two variables. If $c_{i}$ is of assertive type, the arc function from the place representing $r_{i}$ is same as $x$ in $f_{2}$, otherwise if $c_{i}$ is of exclusive type, it will be a different variable than $x$. (14) A relative time constraint $d$ between two tasks can be associated as a delay $d$ at the constraint place.

Figure 3.1 shows the CTPN representation of the authorization model for a workflow consisting of role $r$, object type $\gamma$ and a task $t w . s_{1} \ldots s_{m}$ are subjects assigned to role $r$ and $o_{1} \ldots o_{n}$ denote objects of type $\gamma$. Ensuring that only objects of specified type and subjects of specified role are assigned a privilege $p r$ is reflected by the input arc functions to the grant transition of $p r$. The granting of an authorization is represented by the firing of $t_{s}$ when an object of type $\gamma$ arrives with an authorized subject from role $r$, thereby starting the execution of $t w$. Then a token of color $(x, o)$ is placed in $p r$. The authorization is thus derived based on the value (or color) and timestamp of the input tokens. Also note that the privilege $p r$ is not granted for $x$ on $o$ until $t_{s}$ fires and a token 


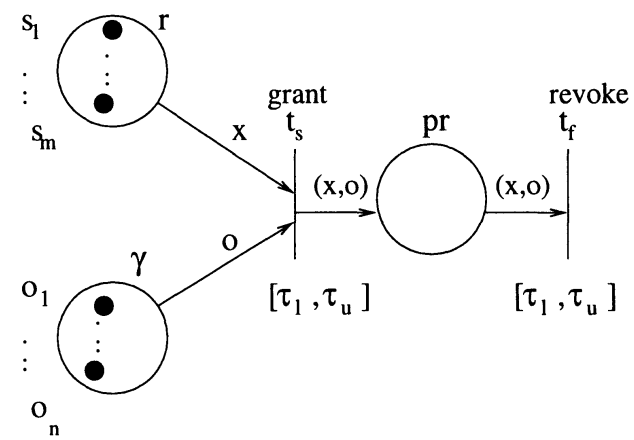

Figure 3.1 A CTPN representation of WAM for a Workflow with one task

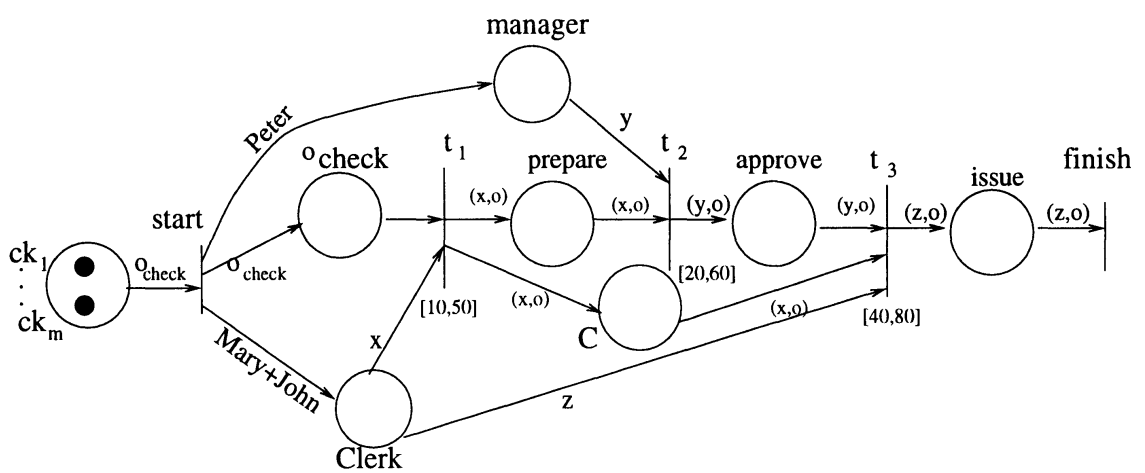

Figure 3.2 A CTPN representation of the check processing example

with $(x, o)$ is deposited in $p r$. The token resides in $p r$ until $t_{f}$ fires (i.e., as long as the task is executed). This firing removes the token from $p r$ thereby revoking the authorization from the subject. Figure 3.2 shows the CTPN representation for the check processing example. The start and finish transitions represent the initiation and completion of each workflow instance.

\subsubsection{Approach to Conduct Safety Analysis}

In this section, we demonstrate how CTPN representation lends itself to address the safety problem of WAM. Our CTPN representation of WAM is such that the safety problem in authorization models is equivalent to the reachability problem in Petri nets. Reachability is a fundamental property for studying the dynamic properties of any system. It can be formally defined as follows.

Definition 15 [9] A marking $M$ is said to be reachable from a marking $M_{0}$ if there exists a sequence of firings that transforms $M_{0}$ to $M$.

Since in our model, authorizations are represented as a marked place $p r$ with token $(s, o)$, the safety question whether $s$ possesses $p r$ on $o$ can simply 
be answered by conducting a reachability analysis on the corresponding CTPN which answers whether a place $p r$ will ever be marked by a token $(s, o)$. Thus,

\section{Safety of WAM $\equiv$ Reachability of its CTPN representation}

since workflow authorization state in WAM is equivalent to marking of its corresponding CTPN.

Therefore, existing reachability analysis techniques, methods and results can be directly adopted to WAM. One may use one of the following three approaches to conduct safety analysis:

1. Simulation: Simulation is a technique to analyze a system by conducting controlled experiments. However, sometimes it is expensive and also it is not possible to use simulation to prove that the system has the desired properties because it is not a formal analysis technique.

2. Reachability tree: The basic idea behind the reachability tree is to construct a graph which contains a node for each reachable state and an arc for each possible change of the state. This is similar to unfolding the maximal state in [11] for analyzing TAM (The Typed Access Matrix Model). Since this represents all possible states, we can answer all safety questions. However, this tree can grow infinitely large even for a small PN (if they are unbounded). It has been shown in [8] that the reachability problem although decidable, has at least exponential space and time complexity.

3. Matrix-equations: In this approach, the dynamic behavior of a $\mathrm{PN}$ is captured in algebraic equations that can be represented as a matrix. Given an initial state, this technique allows us to determine whether a specific state is reachable. However, this requires the $\mathrm{PN}$ to be acyclic.

Fortunately, for our $C T P N$ representation of workflow, we can adopt the less expensive matrix equation approach. Before we present our approach, we first recognize that our CTPN representation of an consistent workflow specification is acyclic in its structure, referred to as acyclic Petri net, which does not contain cycles.

Definition 16 [1] A workflow specification $W$ is said to be inconsistent if either of the following is true: (1) the set of dependencies impose a cyclic execution order among some of the tasks in $W(2)$ the set of dependencies specify that a set of complementary states (e.g., $\mathrm{cm}$ and $a b$ ) have to be reached for at least one task in $W$.

In the above definition, the first condition specifies an inconsistent precedence relationship among the tasks. For example, a set of two dependencies where one dependency states task $t w_{1}$ can begin only after task $t w_{2}$ completes the execution, and the other dependency states $t w_{1}$ has to be executed before $t w_{2}$, would form an inconsistent precedence ordering between $t w_{1}$ and $t w_{2}$. The second requirement specifies inconsistent logical relationships among the task dependencies. For example, if we consider a set of dependencies where one states if $t w_{1}$ aborts, $t w_{2}$ has to abort and the other dependency specifies $t w_{2}$ has to commit if $t w_{1}$ aborts. There is an obvious inconsistency for $t w_{2}$ if $t w_{1}$ 
aborts. Refer to [1] for a detailed discussion on consistency checking. It has been shown in [1] that if the workflow specification is consistent then there does not contain any cycle in the corresponding CTPN representation. This leads to the following proposition.

Proposition 1 If the workflow specification $W$ is consistent, then the corresponding CTPN is acyclic.

Our approach to conducting safety analysis relies on the following theorem.

Theorem 1 [9] In an acyclic Petri net, a marking $M_{i}$ is reachable from an initial marking $M_{0}$, iff there exists a nonnegative integer solution $U$ satisfying

$$
M_{i}=M_{0}+A U,
$$

where $A$ is the corresponding incidence matrix that can be derived from the Petri net as follows: $A$ is an $(m \times n)$ matrix such that $m$ and $n$ are the number of places and transitions, respectively, and each $a_{i j}=a_{i j}^{+}-a_{i j}^{-}$where $a_{i j}^{+}\left(a_{i j}^{-}\right)$ is the number of arcs from transition $j$ to its output (input) place $i$. (See [9] for a proof.)

\section{Algorithm 1 [Safety Analysis of WAM]}

Given a workflow specification $W$, an initial state of authorization and the final state of authorization,

1. Construct $C T P N$ of $W$ according to the mapping in section 3.4.1.

2. Obtain the initial marking $M_{0}$ of $C T P N$ by marking $C T P N$ according to the given initial authorization state.

3. Obtain the final marking $M_{i}$ of $C T P N$ by marking $C T P N$ according to the given final authorization state.

4. Solve $M_{i}=M_{0}+A U$. If $U$ is a non-negative integer, output Yes and stop; otherwise output No.

The above algorithm presents the approach to test for the safety of WAM. The complexity of this algorithm is nothing but that of solving $m$ equations with $n$ variables where $m$ and $n$ represent the number of places and transitions, respectively.

\subsubsection{Implementation}

We have implemented a Workflow Specification and Analysis Software (WSAS) in Visual Basic. WSAS consists of two parts: The first part is to test whether the specification of the workflow is correct or not. The second part tests the safety of workflows, i.e., given an initial state of the workflow, it tests whether a specified state is reachable or not. WSAS has been built on top a prototype, called IDEF/System Dynamics Evaluation Software, developed by Boucher and Jafari [4], which assumes the underlying Petri net model is an ordinary Petri net. Currently we are working on enhancing WSAS to handle CTPN. Our approach is as follows. If the number of subjects is finite, the color sets associated with each place in a CTPN can be converted into a finite number of parallel arcs represented by an ordinary PN. By doing so, our WSAS can be utilized for conducting the analysis. 


\subsection{CONCLUSIONS}

In this paper, we have shown how authorization constraints representing separation of duties can be incorporated into workflow authorization models. We have proposed a methodology to conduct safety analysis of workflow authorization models. We are currently extending the tool which we have developed to test for safety of workflows to address the safety of WAM. Since our software tool can only analyze ordinary Petri nets, we are currently investigating translating the CTPN to an ordinary PN so that our tool can be directly used to analyze WAM.

\section{References}

[1] N.R. Adam, V. Atluri, and W. Huang. Modeling and Analysis of Workflows Using Petri Nets. Journal of Intelligent Information Systems, 10(2), 1998.

[2] V. Atluri and W. Huang. An Authorization Model for Workflows. In Proc. of the Fifth European Symposium on Research in Computer Security, September 1996.

[3] E. Bertino, E. Ferrari, and V. Atluri. A Flexible Model Supporting the Specification and Enforcement of Role-based Authorizations in Workflow Management Systems. In Proc. of the 2nd ACM Workshop on Role-based Access Control, November 1997.

[4] T.O. Boucher and M.A. Jafari. Feasibility of integrating IDEF methodology with testing of system dynamics. Technical Report STP\#24, Rutgers University, The Center for Advanced Food Technology, New Brunswick, NJ, July 1996.

[5] D. Georgakopoulos, M. Hornick, and A. Sheth. An Overview of Workflow Management: From Process Modeling to Workflow Automation Infrastructure. Distributed and Parallel Databases, pages 119-153, 1995.

[6] M.H. Harrison, W.L. Ruzzo, and J.D. Ullman. Protection in Operating Systems. Communications of the ACM, 19(8), 1976.

[7] K. Jensen. Color petri nets: A high level language for system design and analysis. In K.Jensen and G. Rozenberg, editors, High-level Petri Nets Theory and Application, pages 44-119. Springer-Verlag, Lecture Notes in Computer Science, 1991.

[8] S. R. Kosaraju. Decidability and reachability in vector addition systems. In Proc. of the 14th ACM Symposium on Theory of Computing, pages 267-281, May 1982.

[9] T. Murata. Petri nets: Properties, analysis and applications. Proceedings of the IEEE, 77(4):541-580, April 1989.

[10] R. S. Sandhu. Separation of Duties in Computerized Information Systems. In Sushil Jajodia and Carl Landwehr, editors, Database Security, IV: Status and Prospects, pages 179-189. North Holland, 1991. 
[11] R. S. Sandhu. The Typed Access Matrix Model. In Proc. IEEE Symposium on Security and Privacy, pages 122-136, May 1992. 